HEART REVIEW

\title{
Non-cardiac comorbidities in chronic heart failure
}

\author{
Chim C Lang, Donna M Mancini
}

Non-cardiac comorbidity complicates heart failure care and is prevalent in one form or another for the majority of elderly patients with heart failure. This wide range of comorbidities, which includes respiratory comorbidities, renal dysfunction, anaemia, arthritis, cognitive dysfunction and depression, contributes to the progression of the disease and may alter the response to treatment. Polypharmacy is inevitable in these patients. Cardiologists and other physicians caring for patients with chronic heart failure (CHF) need to be vigilant to comorbid conditions that may complicate the care of these patients. Future trials should focus on optimal strategies for the comprehensive management of the elderly patients with CHF with multiple comorbidities rather than the isolated effects of single drugs in younger patients with few or no comorbidities.

See end of article for authors' affiliations

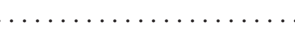

Correspondence to: Professor C C Lang Department of Cardiology, Ninewells Hospital and Medical School, Dundee DDI 9SY, UK;

c.c.lang@dundee.ac.uk

Accepted 30 January 2006 Published Online First 17 February 2006

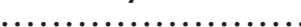

Heart 2007;93:665-671. doi: 10.1136/hrt.2005.068296

Medicare elderly patients aged $\geqslant 65$ years, hospitalised with a principal diagnosis of CHF. ${ }^{3}$ About a third $(32.9 \%)$ had chronic obstructive pulmonary disease (COPD), $18 \%$ had a history of stroke and $9.2 \%$ had dementia. More recently, Braunstein and colleagues $^{4}$ reported the findings of a crosssectional analysis of 122630 individuals aged $\geqslant 65$ years with CHF identified through a 5\% random sample of all US Medicare beneficiaries. Nearly $40 \%$ of patients with CHF had $\geqslant 5$ noncardiac comorbidities, and this group accounted for $81 \%$ of the total inpatient hospital days experienced by patients with CHF. The top 10 most common non-cardiac conditions were COPD/ bronchiectasis $(26 \%)$, osteoarthritis $(16 \%)$, chronic respiratory failure or other lower respiratory disease excluding COPD/bronchiectasis (14\%), thyroid disease (14\%), Alzheimer's disease/dementia $(9 \%)$, depression $(8 \%)$, chronic renal failure $(7 \%)$, asthma (5\%), osteoporosis $(5 \%)$ and anxiety (3\%). The risk of hospitalisation and potentially preventable hospitalisations strongly increased with the number of chronic conditions (fig 1). After controlling for demographic factors and other diagnoses, comorbidities that were associated consistently with higher risks for $\mathrm{CHF}$ hospitalisations and mortality included COPD/ bronchiectasis, renal failure, diabetes, depression and lower respiratory diseases. Several reasons may explain why older patients with CHF with greater comorbidity may experience more adverse events that lead to preventable hospitalisations. These include underutilisation of effective CHF treatments in the presence of other conditions because of safety concerns (eg, use of $\beta$-blockers in asthma or ACE inhibitors in renal insufficiency), patient non-adherence to or inability to recall complex medication regimens, inadequate postdischarge care, failed social support and failure to promptly seek medical attention during symptom recurrence. Psychological stress from chronically poor health may also predispose to bad outcomes. Finally, elderly patients with multiple comorbidities and polypharmacy are also susceptible to poor coordination of care and are also at an increased risk for experiencing adverse drug reactions from drug-drug interactions. The association between comorbidity and healthcare costs has also been examined in a Medicare healthcare expenditure study. ${ }^{5}$ Patients with CHF having expensive

Abbreviations: $B N P$, brain natriuretic peptide; $\mathrm{CHF}$, chronic heart failure; COPD, chronic obstructive pulmonary disease; CPAP, continuous positive airway pressure; CSA, central sleep apnoea; HF, heart failure; NYHA, New York Heart Association; OSA, obstructive sleep apnoea; rHuEPO, recombinant human erythropoietin; SDB, sleep-disordered breathing; TNF, tumour necrosis factor 


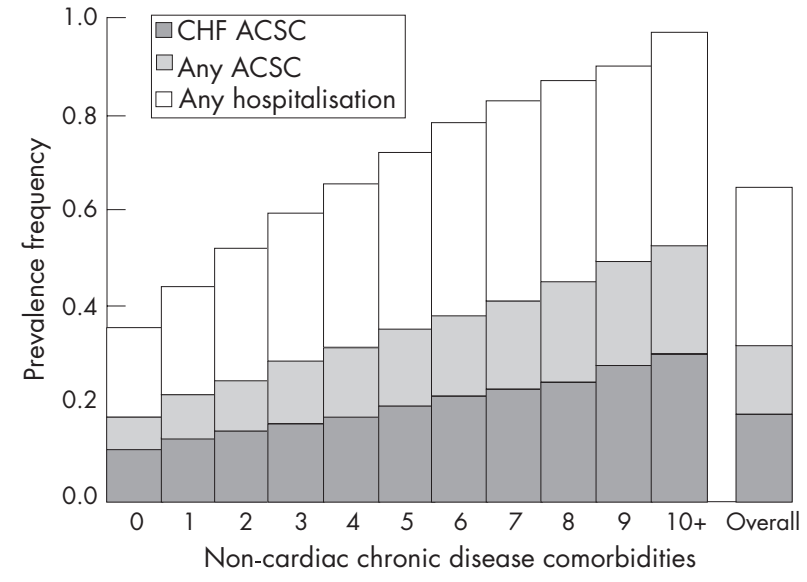

Figure 1 Impact of non-cardiac comorbidity burden on the annual probability of a Medicare beneficiary with chronic heart failure (CHF) experiencing a hospitalisation due to any cause, a preventable hospitalisation or a preventable hospitalisation due to CHF. $p<0.001$ for linear trend for all outcomes. ACSC, ambulatory care sensitive conditions. Reprinted with permission from Braunstein JB, Anderson GF, Gerstenblith $G$, et al. Noncardiac comorbidity increases preventable hospitalizations and mortality among medicare beneficiaries with chronic heart failure. J Am Coll Cardiol 2003;42:1226-33, copyright 2003, with permission from The American College of Cardiology Foundation. ${ }^{4}$

comorbidities included those with chronic pulmonary disease (33\% of patients, mean total annual expenditure \$23104 ( $£ 13355, € 17442$ ) per patient), renal disease (8\% of patients, mean total annual expenditure \$33014 (£19 083, €24 924) per patient), rheumatological disease ( $5 \%$ of patients, mean total annual expenditure, \$20 527 (£11 865, €15 497) per patient) and dementia ( $15 \%$ of patients, mean total annual expenditure, $\$ 26263$ (£15 181, €19 827) per patient).

\section{MANAGEMENT OF SPECIFIC NON-CARDIAC COMORBIDITIES}

We shall now discuss specifically how best to manage CHF in patients with the following common non-cardiac comorbidities in CHF: (1) respiratory comorbidities; (2) renal dysfunction; (3) anaemia; (4) cognitive dysfunction; (5) depression; (6) arthritis.

\section{RESPIRATORY DISORDERS}

The interaction between CHF and concomitant respiratory disease is common and important. Many patients with CHF are misdiagnosed as having air flow obstruction on the basis of overlapping symptoms (and vice versa). In patients presenting to the casualty department with dyspnoea, both brain natriuretic peptide (BNP) and N-terminal prohormone BNP have been shown to be useful in improving the diagnostic accuracy of CHF. ${ }^{6}$ Recently, in a controlled study of the use of both BNP and N-terminal prohormone in 306 patients with suspected CHF referred to rapid access CHF clinics, ${ }^{7}$ both tests proved useful, in particular when the results were normal. When both CHF and respiratory disorders coexist, it may be important to quantify the relative contribution of cardiac and pulmonary components to the disability. Exercise testing with simultaneous gas exchange or blood gas measurements may be helpful in this regard. Optimum assessment and management of these patients need careful consideration of the possibility that cardiac and respiratory disease may coexist in the individual patient.

\section{Obstructive airways disease}

$\beta$-blockers are deemed to be contraindicated in patients with $\mathrm{CHF}$ and air flow obstruction. In practice, because of the overwhelming benefits of these agents in CHF, many patients with fixed or limited airways reversibility are given them, and they tolerate them surprisingly well. Whether $\beta$-l-selective agents offer advantages over non-selective agents such as carvedilol is not clear. ${ }^{89}$ Cardioselective $\beta$-blockers given in mild to moderate reversible airway disease do not produce adverse respiratory effects in the short term. Given their demonstrated benefit in heart failure (HF), these agents should not be withheld from such patients, but long-term safety (especially, their impact during an acute exacerbation) still needs to be established.

\section{Sleep-disordered breathing}

There has been increasing interest in the role of sleepdisordered breathing (SDB) in patients with CHF. Although often neglected in the clinical practice and $\mathrm{CHF}$ literature for many years, SDB in patients with CHF has recently been gaining recognition on the basis of its clinical relevance ${ }^{10}{ }^{11}$ and the subject has recently been reviewed by Cormican and Williams. ${ }^{10}$ It is of significance that both CHF and SDB constitute multisystem disorders involving respiratory, cardiovascular and neurohumoral axes. Two major types of SDB observed in CHF are obstructive sleep apnoea (OSA) and central sleep apnoea (CSA). These two types of SDB operate through different pathophysiological mechanisms, although they can coexist and interact. ${ }^{10}$ OSA results from complete or partial collapse of a normal pharynx. Struggling to breathe against the throat causes generation of negative intrathoracic pressure, leading to marked loading of the ventricles. By contrast, CSA results from either a reduction in central respiratory drive or instability in feedback control of the central respiratory centre. OSA, a likely risk factor for cardiovascular disease, may contribute to both the development and progression of CHF. Moreover, OSA shares with CHF many aspects of deranged neurohumoral and immunological function. CSA, on the other hand, may be a consequence of CHF, but when present, may increase the risk of arrhythmias and impair prognosis. ${ }^{10}$

The prevalence of SDB is high in CHF. Up to $40 \%$ of patients with CHF have CSA and $11 \%$ have OSA. ${ }^{10}$ In the most recent data, SDB is common even in stable outpatients with CHF. ${ }^{12}$ It should be noted that the majority of patients with CHF having SDB remain undiagnosed. This is due to both a limitation in awareness as well as the limited availability of sleep laboratories. Although attended nocturnal polysomnography in a sleep laboratory is the preferred diagnostic method, ${ }^{13}$ homebased unattended sleep studies that combine continuous pulse rate and recording the oxyhaemoglobin saturation have been used as screening tools. ${ }^{10}$ It has been suggested that patients with CHF should also be routinely asked simple screening questions such as "Do you snore?" or "Do you fall asleep during the day?" to help identify potential patients with SDB.

\section{Treatment of SDB}

Haemodynamic improvement following medical treatment is often associated with a significant improvement in the nocturnal breathing pattern. In patients with persistent SDB, especially when it is associated with marked desaturations and refractory $\mathrm{CHF}$, a specific treatment of the breathing disorder should be considered.

\section{Nocturnal nasal continuous positive airway pressure} There have been a number of randomised controlled trials of continuous positive airway pressure (CPAP) therapy in patients with CHF and OSA or CSA. In terms of OSA, there have been at 
least four small-scale studies, ${ }^{10}$ which consistently show that CPAP reverses obstructive apnoea and improves oxygenation at night. CPAP also lowers sympathetic activity and improves left ventricular ejection fraction of up to $9 \%$. There are a similar number of randomised controlled trials of CPAP in CSA when applied for at least 1-3 months with similar benefits of reduced apnoea-hypopnoea index, improvement in ejection fraction $(6.5-8.6 \%)$ and functional class. ${ }^{10}$ It should be emphasised that the sample sizes of these trials are small and of short follow-up and consequently, it was only the study by Sin and colleagues ${ }^{14}$ that reported a risk reduction in combined mortality-cardiac transplantation rate in CHF with CSA but not in patients without CSA. Nevertheless, these findings prompted the Canadian Continuous Positive Airway Pressure trial, the results of which have just been published. ${ }^{15}$ The study was terminated early in May 2004 when there was a dramatic drop in the expected rate of primary events caused largely by the introduction of $\beta$-blockers. This resulted in the study being statistically underpowered. Only 258 of the anticipated 408 patients were randomised with a mean follow-up of 24 months. Despite a reported $50 \%$ reduction in the apnoea-hypopnoea index (39.7-18.6 apnoeas per hour, $\mathrm{p}<0.001$ ), an improvement in both left ventricular systolic function $(p=0.007)$, and an increase in 6-minute walking distance (an increase of $20 \mathrm{~m}$ vs $-0.8 \mathrm{~m}, \mathrm{p}=0.016$ ), the transplant-free survival and rates of CHF-related hospitalisations were identical in the CPAP and control groups. Of concern was the observation of an early divergence of events favouring the control group during the first 18 months $(p=0.02)$, which then reversed and favoured the CPAP group after 18 months. The mechanism for this observation is a subject of debate and has not been fully defined. Thus, although CPAP alleviated CSA and improved cardiac function, its effectiveness in improving transplant-free survival remains in question.

\section{OTHER TREATMENT STRATEGIES FOR SDB IN CHF}

The adaptive servo-ventilator has been designed for the treatment of CSA in patients with CHF and provides a baseline degree of ventilatory support, where the patient's ventilation is servo-controlled to maintain the ventilation at $90 \%$ of the longterm average. ${ }^{16}$ In a crossover trial conducted in 14 patients in New York Heart Association (NYHA) II-III, this approach provided an additional $83 \%$ reduction in central apnoeas when compared with nasal CPAP, and was also more easily tolerated.

Oxygen supplementation has been tested in CSA although the rationale is not entirely clear. It has been hypothesised that $\mathrm{O}_{2}$ may stabilise the breathing by removing any enhancement of the hypercapnic chemoreflex and attenuate any independent influence of hypoxic chemoreflex activation on the hyperventilatory response. In a crossover study of patients with CHF in NYHA functional class IV, single-night treatments with $\mathrm{O}_{2}$ supplements and nasal CPAP were equally effective in decreasing the apnoea-hypopnoea index, arousal index and degree of desaturation. ${ }^{17}$ Although a clear advantage of $\mathrm{O}_{2}$ is the greater acceptability by the patients, data on the effect of nocturnal $\mathrm{O}_{2}$ treatment on medium-term clinical and functional end points are not currently available.

Interesting results have been shown with oral theophylline, which, in the short term, resulted in a 50\% reduction in apnoea-hypopnoea index and related arousals. ${ }^{18}$ Among the potential mechanisms, an increased inotropic effect and a direct stimulation (and stabilisation) of the central ventilatory drive have been invoked as playing a role in reducing the respiratory events. Data on long-term efficacy and safety are not available at present.

Finally, overdrive pacing has also been shown to elicit a $60 \%$ reduction of both central and obstructive apnoeas in a small group of highly selected patients with symptomatic sinus bradyarrhythmias and coexisting sleep apnoea. ${ }^{19}$ Although interesting, these results should be considered preliminary. Long-term studies are necessary to determine their impact on the natural history of left ventricular systolic dysfunction and on CHF-related morbidity and mortality.

\section{RENAL DYSFUNCTION IN CHF}

The close relationship between cardiovascular and renal function in normal physiology is also apparent in the disease. As a consequence of accelerated atherosclerotic coronary artery disease, concomitant hypertension and fluid retention, patients with primary renal disease are at high risk of CHF. Conversely, many patients with CHF have evidence of kidney dysfunction in the absence of intrinsic renal disease. About $40 \%$ of patients with CHF have chronic kidney disease, defined as a serum creatinine level of $\geqslant 133 \mu \mathrm{mol} / \mathrm{l}$ or a creatinine clearance rate of $<60 \mathrm{ml} / \mathrm{min} .{ }^{20}$ The observed low glomerular filtration rate in CHF is a consequence of diminished cardiac output, with decreased renal perfusion and intrarenal vasoconstriction accompanied by sodium and water retention. Indeed, given the relationship between renal function and cardiac output, renal dysfunction is surprisingly an adverse prognostic marker, and also a strong predictor of poor outcome in HF than functional class. ${ }^{20}$ Multiple theories have been offered to explain the excess risks in patients with CHF with renal dysfunction including more advanced coronary atherosclerosis. However, recent data from 6427 patients with CHF with documented coronary angiography showed that the adverse prognostic influence of renal dysfunction was independent of atherosclerotic burden and left ventricular systolic function. ${ }^{20}$

\section{Heart failure therapy in patients with $\mathrm{CHF}$ with renal dysfunction}

The effects of CHF drugs on patients with CHF with renal dysfunction have not been well studied. Patients with renal hypoperfusion or intrinsic renal disease show an impaired response to diuretics and ACE inhibitors ${ }^{21}$ and are at an increased risk of adverse effects during treatment with digitalis. Most CHF trials have studied patients with normal renal function with only a few reporting subgroup analyses of patients with renal dysfunction. In the Cardiac Insufficiency Bisoprolol Study, those with moderate to severe renal failure showed a similar benefit on mortality and hospitalisation from bisoprolol treatment to those with normal renal function. ${ }^{22}$ Perhaps the most impressive effect of a $\beta$-blocker in CHF and end-stage renal failure was recently reported by Cice et al. ${ }^{23} \mathrm{~A}$ total of 114 dialysis patients with dilated cardiomyopathy were randomised to receive either carvedilol or a placebo in addition to standard therapy. At 2 years, the carvedilol group had smaller cavity diameters in both systole and diastole and had higher ejection fractions. By 2 years, $51.7 \%$ of the patients in the carvedilol group had died, whereas $73.2 \%$ in the placebo group had died. There were significantly fewer cardiovascular deaths and fewer hospital admissions among the patients receiving carvedilol. All these data strongly support the use of such drugs in patients with CHF with chronic kidney disease. In view of the extremely high cardiovascular morbidity and mortality in chronic kidney disease and end-stage renal disease, there is clearly a need for routine use of such cardioprotective agents in patients with cardiac damage. Unfortunately, in the "real patient" population, prescription rates for such CHF drugs are inversely related to renal function. ${ }^{20}$

As the renal vasoconstriction that develops in the setting of reduced cardiac output depends on angiotensin II, treatment with an ACE inhibitor or angiotensin-receptor blocker commonly leads to an (generally, clinically unimportant) increase 
in the serum creatinine concentration. Generally, these slight rises in serum creatinine levels are reversible and are only infrequently the cause of drug discontinuation. Most patients with CHF tolerate mild to moderate degrees of functional renal impairment without difficulty. However, if the serum creatinine increases $>220 \mu \mathrm{mol} / \mathrm{l}$, the presence of renal insufficiency can severely limit the efficacy and enhance the toxicity of established treatments. An arbitrary creatinine cut-off value to define renal insufficiency (serum creatinine $>220 \mu \mathrm{mol} / \mathrm{l}$ ) for spironolactone has been suggested in published guidelines. ${ }^{11}$ However, this may not be appropriate in the elderly because of the competing age-related decline in creatinine as a result of decline in muscle mass and rise in creatinine as a result of decline in glomerular filtration rate. Indeed, in a prescription linked study, Juurlink and colleagues ${ }^{24}$ found that immediately after the publication of the Randomized Aldactone Evaluation Study, the prescription rate in Canada rose sharply and that this was associated with an increase in the rate of admission for hyperkalaemia, from 2.4 per 1000 patients in 1994 to 11 per 1000 patients in $2001(\mathrm{p}<0.001)$, and the associated mortality rose from 0.3 per 1000 to 2 per 1000 patients $(\mathrm{p}<0.001)$. However, it should be noted that there were several differences in this real-patient population study from the cohort in the Randomized Aldactone Evaluation Study trial. These older patients received a higher dose of spironolactone without close attention to serum creatinine and follow-up. Similar findings have also been described elsewhere. In the study of Medicare beneficiaries aged $\geqslant 65$ years discharged after hospitalisation for $\mathrm{CHF}$, spironolactone was prescribed to $22.8 \%$ of patients with serum potassium $\geqslant 5 \mathrm{mmol} / \mathrm{l}$, to $14.1 \%$ with a serum creatinine value $\geqslant 220 \mu \mathrm{mol} / \mathrm{l}$ and to $17.3 \%$ with severe renal dysfunction (glomerular filtration rate $<30 \mathrm{ml} / \mathrm{min} / 1.73 \mathrm{~m}^{2}$ ). ${ }^{25}$ In multivariate analysis, factors associated with such prescribing patterns included advanced age and non-cardiovascular morbidities. Clearly, there is a need for greater vigilance and care to be given to frequent monitoring of electrolytes and renal parameters in patients with CHF with renal dysfunction.

\section{ANAEMIA}

Anaemia has recently been recognised as an important comorbid condition and potentially novel therapeutic target in patients with CHF. Anaemia is common in patients with HF, with a prevalence ranging from $4 \%$ to $55 \%{ }^{26}$ Reasons for this wide variation include differences in the population with CHF studied, in study methods, and in the definition of anaemia used. Although the most commonly accepted definition of anaemia is that of the World Health Organization (haemoglobin $<13 \mathrm{~g} / \mathrm{dl}$ in men and $<12 \mathrm{~g} / \mathrm{dl}$ in women), studies have varied considerably in the criteria used to classify patients as anaemic. In general, the prevalence of anaemia is greater in less-selected populations (such as insurance claims data) and lower in highly selected populations such as patients enrolled in clinical trials. Anaemia appears to be more common in patients with more severe disease, with a reported prevalence in patients with NYHA functional class IV populations as high as $79 \%$. Multiple potential mechanisms of interaction exist between anaemia and the clinical syndrome of HF. CHF is a disease of the elderly, a population where the prevalence of anaemia is high irrespective of cardiac status. Multiple comorbid conditions are common in patients with CHF, in particular, renal insufficiency, which is closely associated with the development of anaemia. Other potential contributing factors include haemodilution, proinflammatory cytokines, malnutrition due to right-sided HF and decreased perfusion to the bone marrow. With respect to haemodilution, expansion of plasma volume is a characteristic of the CHF syndrome, and, therefore, some anaemia may be dilutional rather than due to a true decrease in red blood cell mass. In a study of 37 patients with CHF, we found that, true anaemia (ie, a decrease in red blood cell mass) was present in $54 \%$, and haemodilution was present in $46 \%$. Notably, in this study, both haemodilution and true anaemia were associated with adverse survival, with the worst survival seen in patients with haemodilution. ${ }^{27}$ In reality, it is likely that several of these mechanisms are active simultaneously, and that anaemia in HF is the result of a complex interaction between cardiac performance, neurohormonal and inflammatory activation, renal function and bone marrow responsiveness. A prospective ongoing study including both specialty and community sites, the Study of Anaemia in a Heart Failure Population registry, is evaluating the prevalence, aetiologies and mechanisms of anaemia in a broad population of patients with CHF.

The association of anaemia with adverse clinical outcomes in CHF has led to substantial interest in anaemia as a potential therapeutic target. Preliminary data involving small groups of patients with HF suggest that treatment of anaemia may result in a significant symptomatic improvement in HF. ${ }^{27}{ }^{28}$ We have previously evaluated the effect of 3 months of erythropoietin treatment on exercise capacity in single-blind placebocontrolled study of 26 patients with anaemia and NYHA functional class III-IV HF. ${ }^{28}$ This study demonstrated significant improvements in mean (SD) peak oxygen consumption with erythropoietin treatment (from $11(0.8)$ to $12.7(2.8)$ in the recombinant human erythropoietin (rHuEPO)-treated patients $(\mathrm{p}<0.05)$ vs no significant change in the control patients). A significant correlation was observed between increases in haemoglobin with rHuEPO treatment and increased peak oxygen consumption. Notably, the improvement in exercise performance with rHuEPO treatment was observed whether the anaemia was found to be from decreased red blood cell mass or from haemodilution. These studies used rHuEPO in a regimen similar to the one used in patients with end-stage renal disease. Newer erythropoietin analogs have been developed (such as darbepoetin $\alpha$ ) that have a longer half-life and require less frequent administration, potentially making them more attractive for CHF treatment. It should also be noted that aggressive treatment of anaemia may also be associated with an increased risk of hypertension or thrombosis. Multiple ongoing studies will provide definitive data on the balance of risks and benefits of anaemia treatment in chronic HF. The Study of Anaemia Heart Failure Trial study is an ongoing double-blind, placebo-controlled, randomised trial of about 300 patients with CHF with anaemia (defined as having a haemoglobin level $<12 \mathrm{~g} / \mathrm{dl}$ ) who will be randomised to treatment (subcutaneous injections every 2 weeks for 1 year) with darbepoetin $\alpha$ or placebo. Exercise treadmill tests will be performed at baseline and again at 13 and 27 weeks. Change in functional status will be the main end point.

At present, there is insufficient data to make a general recommendation for aggressive treatment of anaemia in patients with HF. A diagnostic evaluation for potentially reversible causes of anaemia (such as iron deficiency or occult blood loss) and subsequent treatment, if identified, is appropriate in all patients. Although pilot data on the treatment of anaemic patients with HF with erythropoietin analogs are promising, the studies published thus far have been significantly limited by very small sample size, lack of blinding and the use of subjective end points. Treatment of mild anaemia with erythropoietin analogs can, thus, not be considered a proven treatment for CHF based on currently available data, and the results of larger, more carefully controlled clinical trials will be required before such treatment could be considered a viable therapy. 


\section{COGNITIVE DYSFUNCTION}

An abnormal prevalence of cognitive dysfunction ranging from $35 \%$ to $>50 \%$ has been described among patients with CHF. ${ }^{29}$ Reduced cardiac output from CHF may further compromise cerebral blood flow in a patient with borderline perfusion of the cerebrum. Additionally, CHF is largely driven by vascular disease and cerebrovascular disease is an important contributor to multi-infarct dementia. Among older patients with CHF, cognitive dysfunction has been associated with a fivefold increase in the risk of mortality and a sixfold increase in the probability of dependence for the activities of daily living. ${ }^{30}$ Reducing the burden of cognitive dysfunction might allow substantial gains in terms of survival rates, quality of life and resource consumption. However, no interventions are yet known to improve cognitive performance largely because of the incomplete knowledge about the pathophysiology of cognitive dysfunction in these patients. It should be noted that measures of cognitive function have rarely been used in CHF trials, unlike hypertension trials such as Systolic Hypertension in Europe and Study on Cognition and Prognosis in the Elderly. Given the consistent reporting of impaired cognitive function in cross-sectional studies of patients with CHF, perhaps this should be considered as an endpoint for future trials of HF pharmacotherapy.

\section{DEPRESSION AND CHF}

The prevalence rates of depression in patients with CHF range from $13 \%$ to $77 \% .^{31}$ Depression is a graded, independent risk factor for several adverse outcomes, including decreased compliance with treatment recommendations, increases in healthcare costs, hospital admissions and mortality rates. Cognitive-behavioural therapy is the preferred psychological treatment. Cognitive-behavioural therapy emphasises the reciprocal interactions among physiology, environmental events, thoughts and behaviours, and how these may be altered to produce changes in mood and behaviour. Pharmacologically, the selective serotonin reuptake inhibitors are recommended, whereas the tricyclic antidepressants are not recommended for depression in patients with congestive HF. ${ }^{32}$ Consideration should also be given to potential interactions between antidepressant medications and those commonly used in the treatment of CHF. For example, the tricyclics, as well as fluoxetine, paroxetine and sertraline, have been reported to interact with warfarin to increase prothrombin time. The combination of a selective serotonin reuptake inhibitor with cognitive-behavioural therapy is often the most effective treatment.

\section{ARTHRITIS}

In the study by Braunstein and colleagues, ${ }^{4}$ arthritis is one of the top 10 non-cardiac comorbidities in CHF and its treatment influences HF status. Both non-steroidal anti-inflammatory drugs and cyclo-oxygenase-2 selective inhibitors are associated with potentially significant cardiovascular adverse effects in $\mathrm{CHF}^{33}$ Sodium and water retention with these agents may adversely affect volume status partly because of activation of vasodilator prosatglandins such as $\mathrm{E}_{2}$ and $\mathrm{I}_{2}{ }^{33}$ The premature suspension of the Alzheimer's Disease Anti-inflammatory Prevention and the Adenoma Prevention with Celecoxib trials has prompted intense review of the cardiovascular safety profile of cyclo-oxygenase-2 inhibitors. With regards to non-steroidal anti-inflammatory drugs, if treatment cannot be avoided, educating the patient is important and it is recommended that the lowest dose be used and for the shortest duration possible with close monitoring of CHF status and renal function.

The role of the prostaglandin inhibitor, aspirin, in attenuating the beneficial effects of renin-angiotensin blockade in CHF is highly controversial. Although a systematic review of six large ACE inhibitor trials reported a non-significant negative interaction between ACE inhibitors and aspirin $(p=0.007)$, the trial in that systematic review included very few patients with serum creatinine $>175 \mu \mathrm{mol} / \mathrm{l} .{ }^{34}$ On the other hand, in studies that have included patients with moderate or severe renal insufficiency, many of whom were treated with lower doses of ACE inhibitors and higher doses of aspirin than employed in the meta-analysed trials, have reported a negative interaction. ${ }^{20}$ The dose of aspirin may matter. ${ }^{35}$ It should be noted that although the Warfarin and Antiplatelet Therapy in Chronic Heart Failure trial reported excess CHF hospitalisations in aspirin-treated patients $(88 \%$ of whom were taking ACE inhibitors), there were too few patients with moderate or severe renal insufficiency to definitely answer the ACE inhibitor-aspririn interaction question.

Tumour necrosis factor (TNF) may have a multifaceted contribution to the progression of the disease in CHF. Blockade of TNF, now an established therapy for rheumatoid arthritis and other autoimmune disorders, has been studied in patients with established CHF. Neither the TNF-receptor fusion protein etanercept or the monoclonal antibody infliximab resulted in beneficial outcomes in this setting. ${ }^{36}$

\section{COMORBIDITY AND POLYPHARMACY}

In the patient with CHF with complex comorbidities, physicians typically face the challenge of managing not a single condition but multiple conditions requiring multiple drugs. A recent study of the chronic drugs prescribed at discharge to patients in the US aged $\geqslant 65$ years hospitalised for CHF revealed that the mean number of drugs was 6.8, representing 10.1 daily doses (fig 2). ${ }^{37}$ Unfortunately, little evidence is available to guide this inevitable polypharmacotherapy in patients with CHF and multiple comorbidities. Typically, most CHF trials, either implicitly or explicitly, excluded older patients and patients with multiple comorbidities. In addition, some trials implement run-in periods to assess tolerance to regimens-an approach that may constrain the applicability of the results. Given the lack of data, how should one approach polypharmacy in CHF? Collaborative disease management programmes that include the careful review of drug lists have been shown to reduce hospital admission rates and reduce the costs of care. ${ }^{38}$ Whenever possible, patients with CHF with multiple competing comorbidities and polypharmacy, need to be enrolled in such programmes. Regardless of the availability of disease management programmes, clinicians need to have systems in place to review medication lists carefully at every visit of a patient, with the goal of eliminating drugs that are not known to provide a clear benefit. When initiating new medications, particular attention needs to be paid to the possibility of adverse drug interactions. In treating coexisting conditions, many commonly used drugs need to be avoided whenever possible in patients with HF, based on known pharmacological principles and recommendations from guidelines.

\section{CONCLUSIONS}

In conclusion, non-cardiac comorbidity frequently complicates CHF care particularly in elderly patients with CHF. Indeed, these are the patients most likely encountered in clinical practice. Under-representation of patients with comorbidities in large CHF clinical trials makes generalisation of trial findings to these patients somewhat difficult. Clinical research must adapt to ensure its relevance, and trials need to include not just young patients with systolic dysfunction and little comorbidity. Ongoing studies enrolling the often ignored group of elderly patients with preserved systolic function, such as the Perindopril in Elderly People-CHF study, are an encouraging 
1998-1999 ( $n=31602) \square 2000-2001(n=30774)$
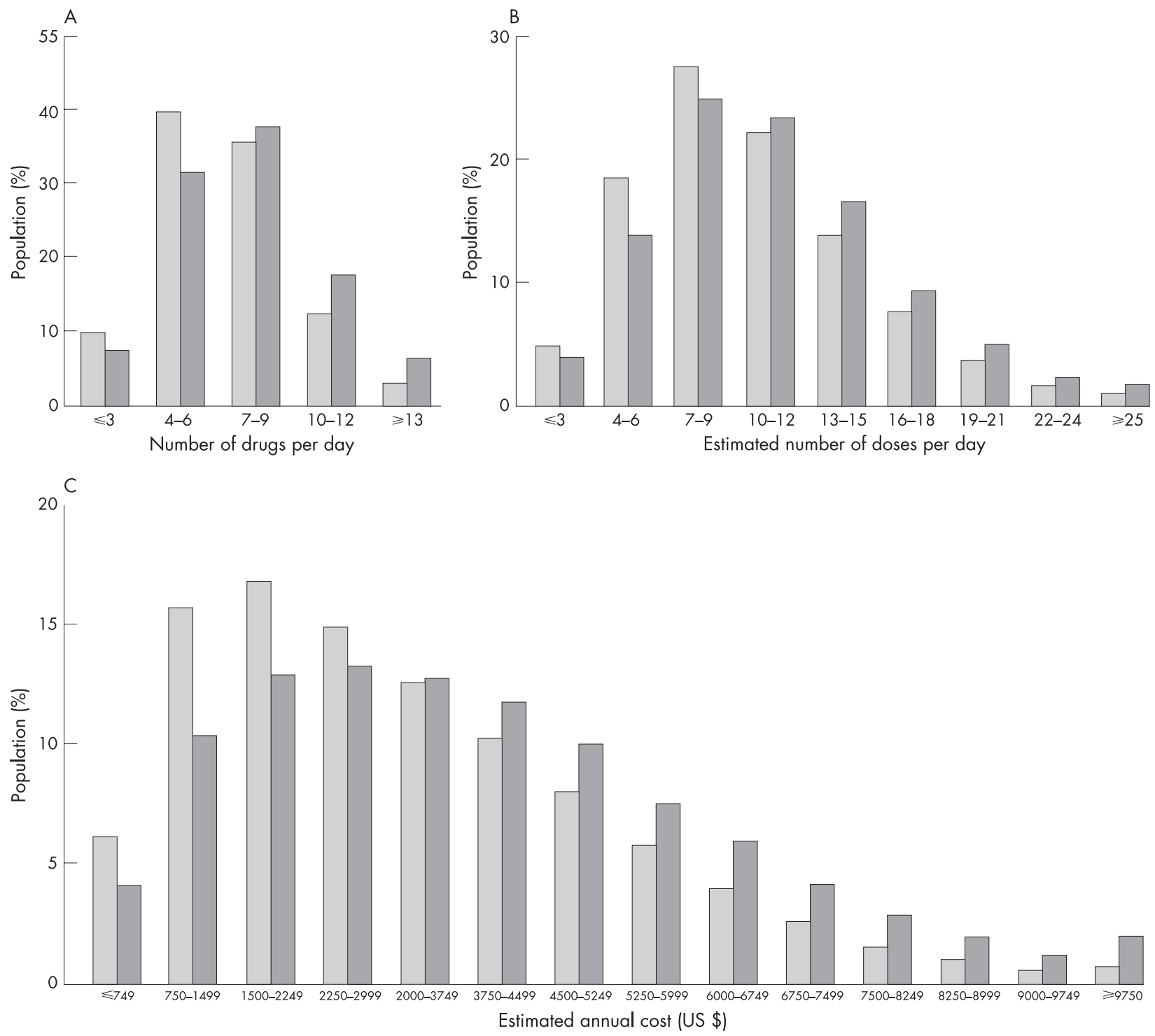

Figure 2 Distribution of the number of long-term prescription medications (A), estimated number of doses per day (B) and estimated annual cost for longterm medications (C) provided at hospital discharge to elderly patients in the US, hospitalised with heart failure. Reprinted with permission from Masoudi FA, Baillie CA, Wang Y, et al. The complexity and cost of drug regimens of older patients hospitalized with heart failure in the United States, 1998-2001. Arch Intern Med 2005; 165:2069-76..$^{37}$ Copyright 2005 American Medical Association.

development but only represent the beginning of a necessary trend. Future trials must also focus on optimal strategies for the comprehensive management of the patient with CHF with multiple comorbidities rather than the isolated effects of single drugs in younger patients with few or no comorbidities.

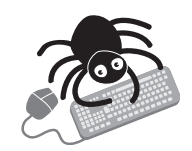

Supplementary references available online at Heart website, http://heart.bmi.com/supplemental

\section{Authors' affiliations}

Chim C Lang, Department of Cardiology, Ninewells Hospital and Medical School, Dundee, UK

Donna M Mancini, Department of Medicine, Columbia University College of Physicians and Surgeons, New York, USA
Competing interests: None.

\section{REFERENCES}

1 Wang TJ, Larson MG, Levy D, et al. Temporal relations of atrial fibrillation and congestive heart failure and their joint influence on mortality: the Framingham Heart Study. Circulation 2003;107:2920-5.

2 Brown AM, Cleland JG. Influence of concomitant disease on patterns of hospitalization in patients with heart failure discharged from Scottish hospitals in 1995. Eur Heart J 1998;19:1063-9.

3 Havranek EP, Masoudi FA, Westfall KA, et al. Spectrum of heart failure in older patients: results from the National Heart Failure Project. Am Heart $J$ 2002; 143:412-17.

4 Braunstein JB, Anderson GF, Gerstenblith G, et al. Noncardiac comorbidity increases preventable hospitalizations and mortality among medicare beneficiaries with chronic heart failure. J Am Coll Cardiol 2003:42:1226-33.

5 Zhang JX, Rathouz PJ, Chin MH. Comorbidity and the concentration of healthcare expenditures in older patients with heart failure. J Am Geriatr Soc 2003;51:476-82

6 Mueller T, Gegenhuber A, Poelz W, et al. Diagnostic accuracy of B-type natriuretic peptide and amino terminal proBNP in the emergency diagnosis of heart failure. Heart 2005;91:606-12. 
7 Zaphiriou A, Robb S, Murray-Thomas T, et al. The diagnostic accuracy of plasma BNP and NT proBNP in patients referred from primary care with suspected heart failure: results of the UK natriuretic peptide study. Eur J Heart Fail 2005; 7:537-41.

8 Salpeter SS, Ormiston T, Salpeter EE. Cardioselective beta-blockers in patients with reactive airways disease: a meta-analysis. Ann Intern Med 2002;137:715-25.

9 Kotlyar E, Keogh AM, MacDonald PS, et al. Tolerability of carvedilol in patients with heart failure and concomitant chronic obstructive pulmonary disease or asthma. J Heart Lung Transplant 2002;21:1290-5.

10 Cormican LJ, Williams A. Sleep disordered breathing and its treatment in congestive heart failure. Heart 2005;91:1265-70.

11 Hunt SA, Abraham WT, Chin MH, et al. ACC/AHA 2005 guidelines update for the diagnosis and management of chronic heart failure in the adult: summary article: a report of the American College of Cardiology/American Heart Association Task Force on Practice Guidelines (Writing Committee to Update the 2001 Guidelines for the Evaluation and Management of Heart Failure). J Am Coll Cardiol 2005:46:1116-43.

12 Ferrier K, Campbell A, Yee B, et al. Sleep-disordered breathing occurs frequently in stable outpatients with congestive heart failure. Chest 2005;128:21 16-22.

13 American Academy of Sleep Medicine Task Force. Sleep related breathing disorders in adults: recommendations for syndrome definition and measurement techniques in clinical research. Sleep 1999:22:667-89.

14 Sin DD, Logan AG, Fitzgerald FS, et al. Effects of continuous positive airway pressure on cardiovascular outcomes in heart failure patients with and without Cheyne-Stokes respiration. Circulation 2000;102:61-6.

15 Bradley TD, Logan AG, Kimoff RJ, et al. for the CANPAP Investigators. Continuous airway pressure for central sleep apnea and heart failure. N Engl J Med 2005;353:2025-33.

16 Teschler H, Döhring J, Wang YM, et al. Adaptive pressure support servoventilation. A novel treatment for Cheyne-Stokes respiration in heart failure. Am J Respir Crit Care Med 2001;164:614-19.

17 Krachman SL, D'Alonzo GE, Berger TJ, et al. Comparison of oxygen therapy with nasal continuous positive airway pressure on Cheyne-Stokes respiration during sleep in congestive heart failure. Chest 1999;116:1550-7.

18 Javaheri S, Parker TJ, Wexler L, et al. Effect of theophylline on sleep-disordered breathing in heart failure. New Engl J Med 1996;335:562-7.

19 Garrigue S, Bordier P, Jais P, et al. Benefit of atrial pacing in sleep apnea syndrome. New Engl J Med 2002;346:404-12.

20 Ezekowitz J, McAlister FA, Humphries $\mathrm{KH}$, et al, for the APPROACH Investigators. The association among renal insufficiency, pharmacotherapy and outcomes in 6,427 patients with heart failure and coronary artery disease. J Am Coll Cardiol 2004;44:1587-92.

21 Philbin EF, Santella RN, Rocco TA Jr. Angiotensin-converting enzyme inhibitor use in older patients with heart failure and renal dysfunction. J Am Geriatr Soc 1999; $47: 302-8$
22 Erdmann $E$, Lechat $P$, Verkenne $P$, et al. Results from post-hoc analyses of the CIBIS II trial: effect of bisoprolol on high-risk patient groups with chronic heart failure. Eur J Heart Fail 2001;3:469-79.

23 Cice G, Ferrara L, D'Andrea A, et al. Carvedilol increases two-year survival in dialysis patients with dilated cardiomyopathy. J Am Coll Cardiol 2003:41:448-54.

24 Juurlink DN, Mamdani MM, Lee DS, et al. Rates of hyperkalemia after publication of the Randomized Aldactone Evaluation Study. N Engl J Med 2004;351:543-5.

25 Masoudi FA, Cross CP, Wang Y, et al. Adoption of spironolactone for older patients with heart failure and left ventricular systolic dysfunction in the United States, 1998-2001. Circulation 2005; 112:39-47.

26 Katz SD. Mechanisms and treatment of anemia in chronic heart failure. Congest Heart Fail 2004; 10:243-7.

27 Androne AS, Katz SD, Lund L, et al. Hemodilution is common in patients with advanced heart failure. Circulation 2003;107:226-9.

28 Mancini DM, Katz SD, Lang CC, et al. Effect of erythropoietin on exercise capacity in patients with moderate to severe chronic heart failure. Circulation 2003:107:294-9.

29 Almeida OP, Flicker L. The mind of a failing heart: a systematic review of the association between congestive heart failure and cognitive functioning. Intern Med J 2001;31:290-5

30 Zuccala G, Pedone C, Cesari $M$, et al. The effects of cognitive impairment on mortality among hospitalized patients with heart failure. Am J Med 2003;115:97-103.

31 Konstam V, Moser DK, De Jong MJ. Depression and anxiety in heart failure. J Card Fail 2005; 11:455-63.

32 Jacob S, Sebastian JC, Abraham G. Depression and congestive heart failure: are antidepressants underutilized? Eur J Heart Fail 2003;5:399-400.

33 Bleumink GS, Feenstra J, Sturkenboom MC, et al. Nonsteroidal antiinflammatory drugs and heart failure. Drugs 2003;63:525-34.

34 Teo KK, Yusuf S, Pfeffer M, et al. Effects of long-term treatment with angiotensinconverting-enzyme inhibitors in the presence or absence of aspirin: a systematic review. Lancet 2002;360:1037-43.

35 Meune C, Mahe I, Mourad JJ, et al. Asprin alters arterial function in patients with chronic heart failure treated with ACE inhibitors: dose-mediated deleterious effect. Eur J Heart Failure 2003;5:271-9.

36 Gullestad L, Aukrust P. Review of trials in chronic heart failure showing broad spectrum anti-inflammatory approaches. Am J Cardiol 2005;95:17C-23C

37 Masoudi FA, Baillie CA, Wang Y, et al. The complexity and cost of drug regimens of older patients hospitalized with heart failure in the United States, 1998-2001. Arch Intern Med 2005;165:2069-76.

38 Rich MW, Beckham V, Wittenberg C, et al. A multidisciplinary intervention to prevent the readmission of elderly patients with congestive heart failure. N Engl J Med 1995;333:1190-5.

\section{The Walter Somerville Award for Medical Writing}

The British Cardiovascular Society and the Editorial Board of Heart are pleased to announce the competition for this annual award, which has been set up as a tribute to the late Walter Somerville in recognition of his 20-year editorship of the British Heart Journal and his enduring interest in the training and mentorship of junior doctors.

The competition is open to any doctor below the rank of consultant or Senior Lecturer and the award will be for the best review article on a clinical cardiovascular subject. The winner will be presented with a certificate at the Annual Scientific Conference and the review will be published. The competition will be judged by the Editor of Heart and the President of the British Cardiovascular Society, who reserve the right not to make the award if none of the entries meet the standard required for publication.

Applications, which must conform with the journal's requirements as published in Instructions to Authors (http://heart.bmj.com/ifora/), together with a statement from the educational supervisor (or equivalent) confirming that the article is the un-assisted work of the candidate, should be submitted electronically (http://submit-heart.bmj.com) by 31 December 2007. 